\title{
Gas-Phase Oligosaccharide Nonreducing End (GONE) Sequencing and Structural Analysis by Reversed Phase HPLC/Mass Spectrometry with Polarity Switching
}

\author{
Xiaoyu Chen* and Gregory C. Flynn \\ Process and Product Development, Amgen, Inc., Thousand Oaks, California, USA
}

Here we describe a technique to obtain all the N-linked oligosaccharide structures from a single reversed-phase (RP) HPLC run using on-line tandem MS in both positive and negative ion modes with polarity switching. Oligosaccharides labeled with 2-aminobenzamide (2AB) were used because they generated good ionization efficiency in both ion polarities. In the positive ion mode, protonated oligosaccharide ions lose sugar residues sequentially from the nonreducing end with each round of MS fragmentation, revealing the oligosaccharide sequence from greatly simplified tandem MS spectra. In the negative ion mode, diagnostic ions, including those from cross-ring cleavages, are readily observed in the $\mathrm{MS}^{2}$ spectra of deprotonated oligosaccharide ions, providing detailed structural information, such as branch composition and linkage positions. Both positive and negative ion modes can be programmed into the same LC/MS experiment through polarity switching of the MS instrument. The gas-phase oligosaccharide nonreducing end (GONE) sequencing data, in combination with the diagnostic ions generated in negative ion tandem MS, allow both sequence and structural information to be obtained for all eluting species during a single RP-HPLC chromatographic run. This technique generates oligosaccharide analyses at high speed and sensitivity, and reveals structural features that can be difficult to obtain by traditional methods. (J Am Soc Mass Spectrom 2009, 20, 1821-1833) (c 2009 American Society for Mass Spectrometry

G lycosylation is one of the most common and complex post-translational modifications of proteins. Differences in carbohydrate moieties can influence the properties and functions of glycoproteins, and serve as a biomarker for disease states [1]. Proper structural determination is thus critical to the understanding of the biological role of carbohydrates. Although there are fewer monosaccharide building blocks in N-linked glycans than there are amino acid moieties in proteins, the combination of multiple branchings and linkages add greater complexity to the glycan structures. Such structural diversity also adds to the difficulty of structural characterization.

Many analytical tools are now available for oligosaccharide characterization, such as high field NMR, liquid chromatography, capillary electrophoresis, and mass spectrometry. Although unambiguous oligosaccharide structural assignments can be made by NMR [2-5], this technique often requires large quantities of highly purified material. Typically, more than $50 \mathrm{mg}$ of glycoprotein and extensive preparative fractionation are re-

Address reprint requests to Dr. X. Chen, Novartis Biologics, 200 Technology Square, Cambridge, MA 02139, USA. E-mail: xiaoyu-1.chen@novartis. com

* Current address: Novartis Biologics, 200 Technology Square, Cambridge, MA 02139, USA. quired to resolve oligosaccharide structures of relatively low abundance. A more common characterization approach utilizes sets of specific exoglycosidases, which cleave terminal monosaccharides from the nonreducing end, followed by chromatographic or MS analysis [6-8]. In this approach, the sequence of the oligosaccharide chain is revealed, either through sequential removal of monosaccharides from the chain upon successive enzyme treatment, or from the oligosaccharide ladder generated upon treatment with an array of enzymes. Linkage positional information and anomericity of the terminal monosaccharide can also be obtained, based on the specificity of the exoglycosidases used. Unfortunately, this classic sequencing technique is often labor intensive, time consuming, and can be limited by the availability of specific enzymes. Tandem mass spectrometry (MS), an approach commonly used for protein/peptide characterization, is becoming more frequently used as an alternative to the classical approaches described [9].

Fragmentation of oligosaccharides in tandem MS experiments yields two major types of cleavages: ones at the glycosidic bonds between two sugar residues and cross-ring cleavages breaking two bonds within a sugar residue. Glycosidic cleavages are prominent under postsource decay (PSD) or low-energy collision-induced dissociation (CID) conditions. This type of cleavage pro- 
vides information mainly on the oligosaccharide sequence but not on the nature of the linkage, although the intensity ratios of certain fragment ions can sometimes be used to differentiate structural isomers [10-12]. One exception is glycosidic cleavages of permethylated glycans [13]. Upon fragmentation, the hydroxyl group involved in a glycosidic bond carries a fragmentation scar of a 14 Da mass difference from other methylated hydroxyl groups, and the terminal, internal, or branching position of the monosaccharide can be determined from the number of scars it has. This approach, however, requires permethylation, and has not been widely used with online LC/MS ${ }^{n}$ analysis. Structurally relevant information may require multi-stage fragmentations with judicious choice of the parent ions at each stage. Cross-ring cleavages, on the other hand, can provide linkage information, and structural isomers can be distinguished by fragment ions that differ in mass rather than relative intensity. In the positive ion mode, metal cationized oligosaccharide ions were found to produce cross-ring cleavages at higher abundance than protonated ions [11, 14]. However, extensive cross-ring cleavages were observed for sodiated ions only under high-energy collision conditions $(\sim 1 \mathrm{keV}$ or higher) [15-25]. In the negative ion mode, cross-ring cleavage was found to be abundant for either deprotonated or anion adduct ions under both high [24] and low [26-36] energy CID conditions. Many diagnostic ions have been reported using these techniques that elucidate detailed oligosaccharide structures at high speed and sensitivity. The interpretation of the tandem MS spectra, unfortunately, is far less straightforward than the classic exoglycosidase sequencing technique.

Efforts to develop online liquid chromatography tandem MS methods for oligosaccharide characterization continue. The LC separation and MS ionization, however, are often compromised, and only limited oligosaccharide structural details were obtained in many cases. Some linkage information can be derived for small or linear oligosaccharides [37-39], whereas spectral matching is utilized to identify more complex glycan structures [40-45]. Enriched structural information was obtained in online pseudo $\mathrm{MS}^{3}$ analysis of permethylated oligosaccharides on a Q-TOF instrument [46]. TOF/TOF or Q-TOF mass spectrometers have been widely used in tandem MS characterization of oligosaccharides. In contrast to such tandem-in-space types of instruments, where tandem MS is limited to a $\mathrm{MS}^{2}$ stage (not including in-source fragmentation), ion storage devices, such as ion trap mass spectrometers, allow multi-stage activations of the parent oligosaccharide ions into successive fragment ions [13, 47-49]. Sequential fragmentation, analogous to successive enzyme treatment in the classic sequencing technique, is thus possible with ion trap instruments.

We report here an on-line RPLC/MS ${ }^{n}$ method for the detailed characterization of oligosaccharides. In this method, N-linked glycans are labeled with 2AB [50]. Fluorescence derivatization with $2 \mathrm{AB}$ is a common approach in carbohydrate analysis [51], including struc- tural characterization by MS (see, for example, Reference [52]). Glycans labeled with 2AB demonstrate good on-line MS signal in both positive and negative ion modes. When coupled with an ion trap instrument, a series of tandem MS experiments can be conducted as oligosaccharide peaks elute during chromatographic separation. In the positive ion mode, sequential loss of sugar residues starting from the nonreducing end of the oligosaccharide chain can be achieved under appropriate collision conditions. This gas-phase oligosaccharide nonreducing end (GONE) sequencing technique provides a simple readout of the oligosaccharide sequence, like the traditional sequencing technique, but with the high speed and sensitivity inherent to the MS technique. In the negative ion mode, structurally diagnostic ions, including those from cross-ring cleavages, are observed in reasonable abundances. Tandem MS spectra from both positive and negative ion modes can be obtained from a single experiment by switching the polarity of the mass spectrometer, thus yielding both sequence and structural information of the oligosaccharide.

\section{Experimental}

\section{Materials and Reagents}

Ribonuclease B (RNase B) from bovine pancreas, ovalbumin (Grade VII) from chicken egg, fetuin from fetal calf serum, $2 \mathrm{AB}$, and sodium cyanoborohydride $\left(\mathrm{NaCNBH}_{3}\right)$ were purchased from Sigma-Aldrich (St. Louis, MO, USA). The glycoproteins were analyzed directly without further purification. Peptide-N-glycosidase F (PNGase F) and reaction kits were obtained from QA-Bio, Palm Desert, CA. Other reagents used were of HPLC reagent grade.

\section{Preparation of 2AB-Derivatized Oligosaccharides}

N-linked oligosaccharides were released enzymatically using PNGase F following the recommended protocol from the manufacturer. Briefly, $200 \mu \mathrm{g}$ glycoproteins in $0.1 \%$ SDS, $50 \mathrm{mM} \beta$-mercaptoethanol, and $50 \mathrm{mM}$ Tris buffer ( $\mathrm{pH}$ 7.5) were denatured by heating at $95^{\circ} \mathrm{C}$ for $5 \mathrm{~min}$. The denatured protein solution was then treated with $0.01 \mathrm{U}$ PNGase $\mathrm{F}$ in the presence of $0.7 \%$ Triton $\mathrm{X}-100$. The digestion was conducted at $37^{\circ} \mathrm{C}$ for $16-24$ $\mathrm{h}$. The resulting digests were loaded onto a PGC column (Alltech, Deerfield, IL) prewashed with $2 \times 1 \mathrm{~mL}$ $0.1 \%$ TFA in $80 \%$ acetonitrile and $3 \times 2 \mathrm{~mL}$ water. The column was then washed with $2 \times 1 \mathrm{~mL}$ water, and the $\mathrm{N}$-glycans were eluted with $2 \times 1 \mathrm{~mL} 0.05 \%$ TFA in $25 \%$ acetonitrile. Eluted fractions were collected and brought to complete dryness using a Speedvac (Genevac, Valley Cottage, NY).

Ten $\mu \mathrm{L}$ labeling reagent $\left(0.35 \mathrm{M} 2 \mathrm{AB}, 1 \mathrm{M} \mathrm{NaCNBH}_{3}\right.$ in 70:30 (vol:vol) DMSO:acetic acid) was added to the released and purified oligosaccharide samples. The reaction mixtures were incubated at $65^{\circ} \mathrm{C}$ for $2 \mathrm{~h}$. The excess labeling reagent was removed by an S-cartridge (QA- 
Bio). The cartridge was first washed with $1 \mathrm{~mL}$ water, then $1 \mathrm{~mL} \mathrm{30 \%}$ acetic acid and $1 \mathrm{~mL}$ acetonitrile. The labeling reaction mixture was then loaded on the Scartridge and left at room temperature for $15 \mathrm{~min}$. After washing with $1 \mathrm{~mL}$ acetonitrile and $4 \times 1 \mathrm{~mL} 85 \%$ acetonitrile, the $2 \mathrm{AB}$ labeled $\mathrm{N}$-glycans were eluted with $3 \times 0.5 \mathrm{~mL}$ water. Eluted fractions were collected and brought to complete dryness, followed by reconstitution with water.

\section{Reversed-Phase HPLC}

The 2AB labeled $\mathrm{N}$-oligosaccharides were separated on a C18 octadecylsilyl (ODS) reversed-phase column ( $3 \mu \mathrm{m}, 4$ $\times 250 \mathrm{~mm}$, Thermo Scientific, Waltham, MA, USA). The mobile phase was a mixture of $0.1 \%$ acetic acid in water (A) and $0.1 \%$ acetic acid in 10:90 acetonitrile:water (B). The column was equilibrated with $30 \%$ B. After holding for 5 min in $30 \% \mathrm{~B}$, the $2 \mathrm{AB}$ labeled $\mathrm{N}$-oligosaccharides were eluted using a gradient of $0.33 \% \mathrm{~B} / \mathrm{min}$ for $135 \mathrm{~min}$. The flow rate was $0.2 \mathrm{~mL} / \mathrm{min}$, fluorescence detection was performed with excitation at $330 \mathrm{~nm}$, and emission at 420 nm. RP-HPLC was performed using an Agilent (Santa Clara, CA, USA) Series 1100 binary pump system directly coupled to the mass spectrometer.

\section{Electrospray Mass Spectrometry}

Mass spectrometry experiments were conducted using a linear ion trap mass spectrometer (LTQ XL; Thermo Scientific), equipped with an ESI source coupled directly to the HPLC instrument. LC/MS ${ }^{n}$ experiments were conducted in both positive and negative ion mode through polarity switching in the same analysis. In both ion modes, the capillary temperature was set at $200{ }^{\circ} \mathrm{C}$. In the positive ion mode, the spray voltage was set at $+4.0 \mathrm{kV}$. For the generation of tandem mass spectra, the parent ions were selected with an $\mathrm{m} / \mathrm{z}$ window of 20 , the normalized collision energy was set to $15 \%$, and the activation was conducted at $Q=0.25$ for $100 \mathrm{~ms}$. Each scan contains two microscans with maximum ion injection time of $200 \mathrm{~ms}$. Benefiting from the resolving power of the RP separation, the wide ion isolation window allows more ions to enter the trap without confounding the tandem MS data by multiple ionic species. In the negative ion mode, the spray voltage was set at $-4.0 \mathrm{kV}$. For the generation of tandem mass spectra, the parent ions were selected with an $\mathrm{m} / \mathrm{z}$ window of 2, the normalized collision energy was set to $30 \%$, and the activation was conducted at $\mathrm{Q}=0.25$ for $100 \mathrm{~ms}$. Each $\mathrm{MS}^{2}$ scan contains three microscans with maximum ion injection time of $500 \mathrm{~ms}$. In both ion modes, ions selected for tandem MS in one MS cycle (see Figure 1) were then excluded for the next three cycles using dynamic exclusion, allowing the fragmentation of low abundant ions.

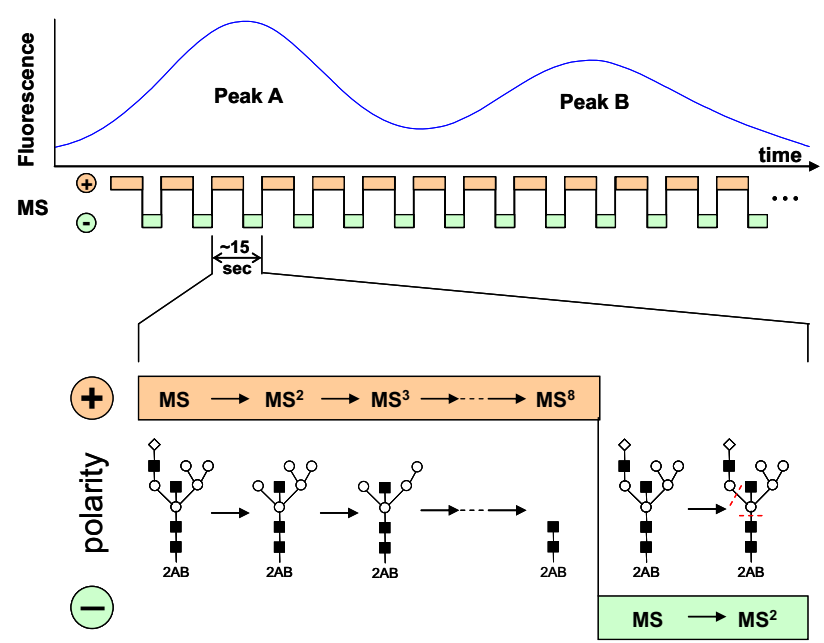

Figure 1. Illustration of the $\mathrm{LC} / \mathrm{MS}^{n}$ experimental scheme. As an example, two peaks (peak A and peak B) from a $2 \mathrm{AB}$ glycan map are shown in the fluorescence chromatogram (top). In the RP separation, the full width at half maximum of each glycan peak is $\sim 1 \mathrm{~min}$, and four or more MS cycles can be performed across each peak. Coeluting species can be analyzed separately in individual MS cycles using dynamic exclusion. The orange box and "+" indicate the positive ion mode. The green box and " - " indicate the negative ion mode. Each MS cycle lasts $\sim 15 \mathrm{~s}$, and consists of multiple orders of $\mathrm{MS}^{n}$ (up to $\mathrm{MS}^{8}$ in the positive ion mode, as well as MS and $\mathrm{MS}^{2}$ in the negative ion mode. The MS scans from a single cycle are illustrated at the bottom, along with hypothetical fragmentations of an example glycan structure. The glycan sequence is revealed through GONE sequencing in the positive ion mode (orange), where sugar residue(s) are sequentially cleaved off from the nonreducing end. Further structure information is obtained from the negative $\mathrm{MS}^{2}$ scan (green). The dashed lines in red indicate the hypothetical cleavages elucidating the glycan structure. The structures shown at each MS stage are for illustration purposes only, and do not imply actual fragmentations. The symbols used are the same as in Figure $2 \mathrm{~d}$, but the connecting lines do not indicate specific linkages.

\section{Results and Discussion}

We have previously reported an online RP-HPLC/MS technique to analyze and characterize $2 \mathrm{AB}$ labeled $\mathrm{N}$-linked oligosaccharides in the positive ion mode [10]. Under similar ion source conditions, the 2AB labeled oligosaccharides showed good ionization efficiency in both positive and negative ion polarities, thereby allowing sequence and structural information to be obtained within a single chromatographic run. In the positive ion mode, the collision conditions were optimized here so that the tandem MS spectrum for each $2 A B$ labeled glycan contained prominently a single $\mathrm{Y}$ type ion [53] resulting from the loss of the nonreducing end sugar residue(s). Upon subsequent fragmentation of the $\mathrm{Y}$ ion, sequential loss of the sugar residue(s) from the nonreducing end was obtained, revealing the sequence of the carbohydrate chain. The secondary amine group on the $2 \mathrm{AB}$ tag is believed to facilitate the generation of those $\mathrm{Y}$ ions by maintaining a charging proton at the reducing end. The mass losses and their corresponding nonreducing end sugar residue(s) in GONE sequencing are summarized in Table 1 . In the negative ion mode, ions 
Table 1. Mass loss defining nonreducing end sugar residue(s) in GONE sequencing

\begin{tabular}{cl}
\hline Mass loss (u, average mass) & Sugar residue(s) \\
\hline \hline 146 & Fuc \\
162 & Man \\
203 & GlcNAc $^{\text {a }}$ \\
291 & SA \\
365 & Gal-GlcNAc \\
527 & Gal-Gal-GlcNAc \\
657 & SA-Gal-GlcNAc
\end{tabular}

${ }^{a}$ Mass loss of $203 \mathrm{u}$ after the loss of one or both core $\alpha$-mannose indicates the presence of bisecting GIcNAc.

diagnostic of the oligosaccharide structure were observed, which are mostly consistent with previous findings under higher collision energies [32] with a few identified in this study. These diagnostic ions, concluded from the negative $\mathrm{MS}^{2}$ spectra of a variety of glycan species (see sections below), are listed in Table 2 to provide guidance for glycan structure elucidation. Both sequence and structural information can be obtained in a single LC/MS experiment by switching the polarity of the MS instrument. The LC/MS experimental scheme is illustrated in Figure 1.

To demonstrate the broad applicability of this method, a variety of different glycan types were analyzed. RNase B, fetuin, and ovalbumin were used because they are known to contain high mannose, complex and hybrid glycans $[2,3,54]$. Since the purpose of this study is to illustrate the utility of the methodology for glycan structure characterization in general, not to provide detailed glycosylation patterns of those glycoproteins, the proteins purchased from commercial sources were analyzed directly without further purification. Figure 2 shows the RP fluorescent chromatographic profiles of $2 \mathrm{AB}$ labeled oligosaccharides released from these glycoproteins. Glycan species containing two or more sialic acids elute earlier than $20 \mathrm{~min}$ and are not included. Although the structures for most of the glycans in the observed peaks have been determined, only peaks labeled in the figure are discussed in this paper, which are representative of different glycan structural features. Solved structures for the labeled peaks are shown in Figure 2d. In N-glycan characterization, biosynthetic restrictions of the expression system are almost always applied, which substantially reduce the number of possible structures. Structures of possible mammalian N-linked complex and high mannose glycans are illustrated in Figure 3. All structures contain a common trimannosyl chitobiose core, attached with a variety of outer sugar residues. The mammalian $\mathrm{N}$ linked hybrid glycans comprise a high-mannose-like 6-antenna and a complex-like 3-antenna, with or without bisecting GlcNAc. For successful characterization of $\mathrm{N}$-linked oligosaccharides, a number of structural features need to be determined, including the compositions of 6- and 3-antennae, the attachment site(s) of fucose and sialic acid, presence of bisecting GlcNAc and $\alpha \mathrm{Gal}$, and differentiation of stereoisomers such as Man and Gal. Characterization results for high mannose, complex, and hybrid glycans from the example mammalian glycoproteins are provided in the following sections to illustrate the approach and to provide diagnostic spectra to guide the identification of unknown glycans. The only structures considered from the MS data were based on mammalian biosynthetic rules as illustrated in Figure 3. The data analysis for an example

Table 2. Diagnostic ions defining $2 \mathrm{AB}$ labeled glycan structures in $\mathrm{MS}^{2}$ spectra of negative ion mode ${ }^{\mathrm{a}}$

\begin{tabular}{|c|c|c|c|}
\hline Diagnostic ions & $m / z^{b}$ & \multicolumn{2}{|c|}{ Glycan structure } \\
\hline$Z_{R}: Y_{R}, Z_{F}$ & $\begin{array}{l}\text { 468:486, M-164 } \\
\text { 322:340, absent }\end{array}$ & $\begin{array}{l}\text { With reducing end fucose } \\
\text { Without reducing end fucose }\end{array}$ & \\
\hline$Z_{N R}: Y_{N R}$ & $\begin{array}{l}M-180: M-162 \\
M-221: M-203 \\
M-309: M-291 \\
M-342: M-324\end{array}$ & $\begin{array}{l}\text { Man or Gal } \\
\text { GIcNAc } \\
\text { SA } \\
\text { Gal-Gal }\end{array}$ & Terminal residue \\
\hline$F\left({ }^{1,3} A_{R-3}\right)$ & $\begin{array}{l}262 \\
424 \\
586\end{array}$ & $\begin{array}{l}\text { GIcNAc } \\
\text { Gal-GIcNAc } \\
\text { Gal-Gal-GIcNAc }\end{array}$ & 1-2,3 linkage \\
\hline$E\left(B^{0,4} X_{R-3}\right)$ & 507 & $(\mathrm{GlcNAc})_{2}$ & 1-2,3,4 linkage \\
\hline B-221 & 3 or $6 \mathrm{~B}-221$ & GlcNAc & 1-4 linkage \\
\hline $\begin{array}{l}{ }_{6} \mathrm{~B},{ }_{6} \mathrm{C},{ }^{0,4} \mathrm{~A}_{\mathrm{R}-2},{ }^{0,3} \mathrm{~A}_{\mathrm{R}-2} \\
\mathrm{D}, \mathrm{D}-\mathrm{H}_{2} \mathrm{O} \\
\mathrm{D}-221, \mathrm{D}-221-\mathrm{H}_{2} \mathrm{O} \\
\mathrm{D}^{\prime}, \mathrm{D}^{\prime}-\mathrm{H}_{2} \mathrm{O}\end{array}$ & $\begin{array}{l}{ }_{6} \mathrm{~B},{ }_{6} \mathrm{~B}+18,{ }_{6} \mathrm{~B}+60,{ }_{6} \mathrm{~B}+90 \\
{ }_{6} \mathrm{~B}+162,{ }_{6} \mathrm{~B}+144 \\
{ }_{6} \mathrm{~B}+144,{ }_{6} \mathrm{~B}+126 \\
{ }_{6} \mathrm{~B}^{\prime}+144,{ }_{6} \mathrm{~B}^{\prime}+126\end{array}$ & $\begin{array}{l}\text { Without bisecting GIcNAc } \\
\text { With bisecting GIcNAc } \\
\text { Outer 6-antenna }\end{array}$ & 6-antenna \\
\hline
\end{tabular}

annotations used in this table and through this paper are based on that of Reference [53] and [32]: superscript before the letter indicates sites of cross-ring cleavages; subscript before the letter indicates 3-or 6-antenna together with the core $\alpha$-mannose to which the antenna is attached; the subscript after the letter indicates the cleavage site, where $R$ and NR represent reducing and non-reducing end respectively, and $F$ represents the cleavage corresponding to the loss of fucose; prime after the letter indicates the 1-6 linked residues on the core $\alpha 1-6$ mannose.

balues for singly charged ions. M denotes molecular ion. 
(a)

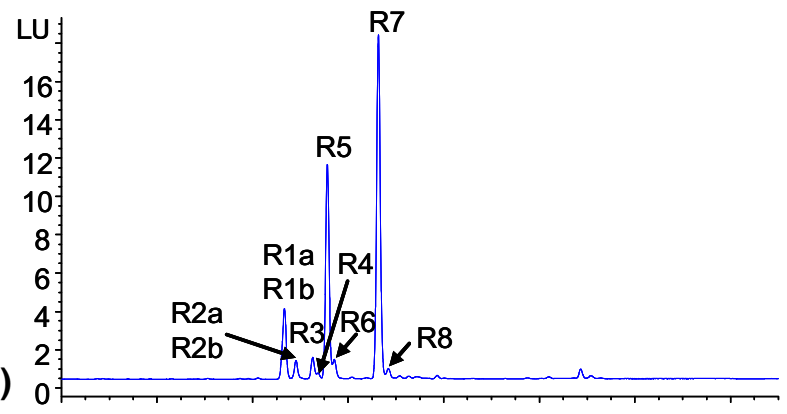

(b)
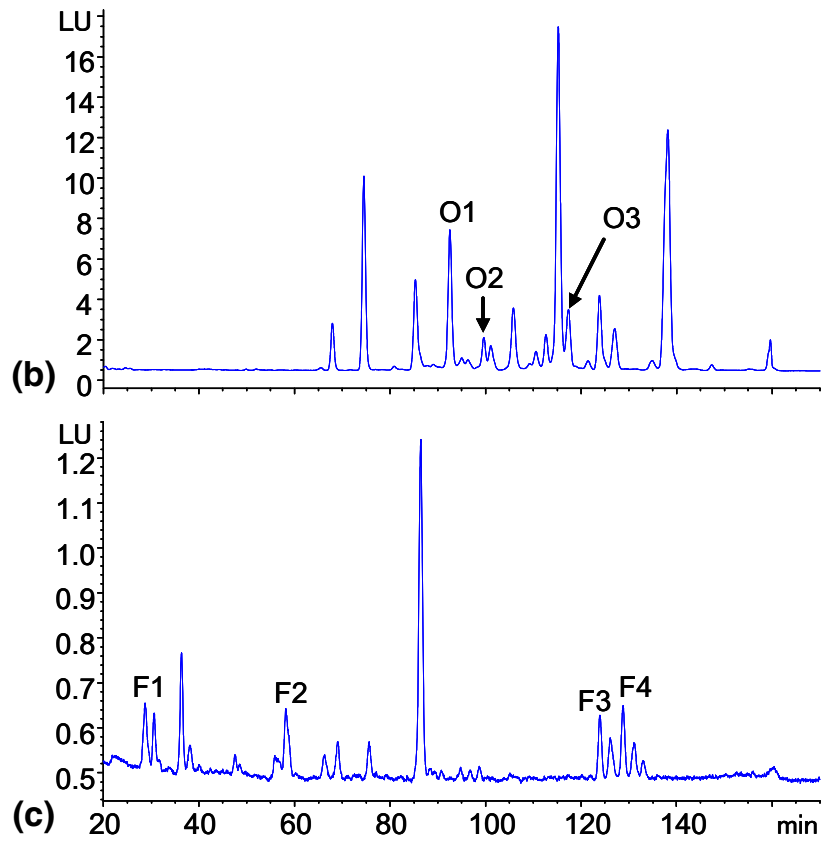

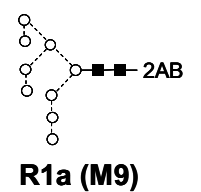

o.

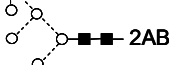

o

R1b (M8-I)

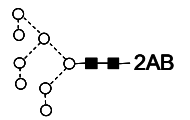

R2a (M8-II)

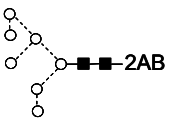

R2b (M7-I)
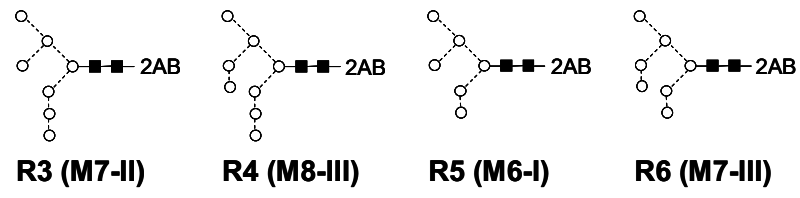

R6 (M7-III)

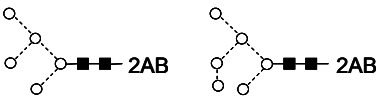

R7 (M5) R8 (M6-II)
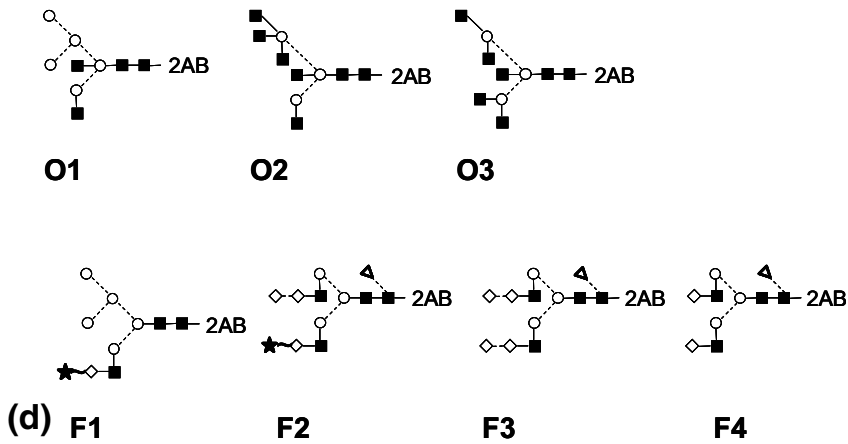

Figure 2. RP Fluorescence chromatograms of the $2 \mathrm{AB}$ labeled glycans released from (a) $10 \mu \mathrm{g}$ RNase B; (b) $30 \mu \mathrm{g}$ ovalbumin; (c) $30 \mu \mathrm{g}$ fetuin. Only oligosaccharide peaks discussed in this paper are labeled, the structures of which are shown in (d). Species co-eluting in the same fluorescent peak are indicated by letters $\mathrm{a}$ and $\mathrm{b}$. The early eluting peaks $(<20 \mathrm{~min})$ containing two or more sialic acids are not shown. Keys to symbols in this figure and subsequent figures: circle, Man; filled square, GlcNAc; diamond, Gal; triangle, Fuc; star, SA; dashed line, $\alpha$ linkage; solid line, $\beta$ linkage; vertical line, 1-2 linkage; horizontal line, 1-4 linkage; forward slash, 1-3 linkage; backward slash, 1-6 linkage; wavy line, unknown linkage.

glycan (F4 in Figure 2) is shown in Figure 3c, demonstrating how the combination of GONE sequencing and negative $\mathrm{MS}^{2}$ data can be used together to determine detailed oligosaccharide structures when biosynthetic restrictions are applied.

\section{High Mannose Glycans}

$2 \mathrm{AB}$ labeled high mannose glycans were prepared from RNase B, the fluorescence RP chromatogram of which is shown in Figure 2a. These high mannose species, including their structural isomers, are well separated by this RP method [10]. The structures of high mannose glycans and their isoforms are shown in Figure 2d, which were determined using on-line tandem MS data. The three structural isoforms [2, 46] of Man7 (peak R2b, R3, and R6) were unambiguously distinguished using tandem MS and are provided as examples for high mannose structural analysis using this technique.

In GONE sequencing, nonreducing end sugar residue(s) are sequentially released in successive $\mathrm{MS}^{n}$ stages, resulting in a single prominent $\mathrm{Y}$ ion in each tandem MS spectrum. This is illustrated in Supplemental Figure S1, which can be found in the electronic version of this article. In the initial MS spectrum $\left(\mathrm{MS}^{1}\right.$, the doubly charged ion at $\mathrm{m} / \mathrm{z} 840$ is the protonated molecular ion of Man7. Upon fragmentation under GONE sequencing conditions, a dominant ion at $\mathrm{m} / \mathrm{z}$ 759 was generated in $\mathrm{MS}^{2}$. The difference in the $\mathrm{m} / \mathrm{z}$ values of those two doubly charged ions is 81 , corresponding to a mass difference of $162 \mathrm{u}$. This mass difference is consistent with the loss of Man from the nonreducing end. Although the mass of Gal is also $162 \mathrm{u}, \mathrm{Gal}$ is always released together with an attached 

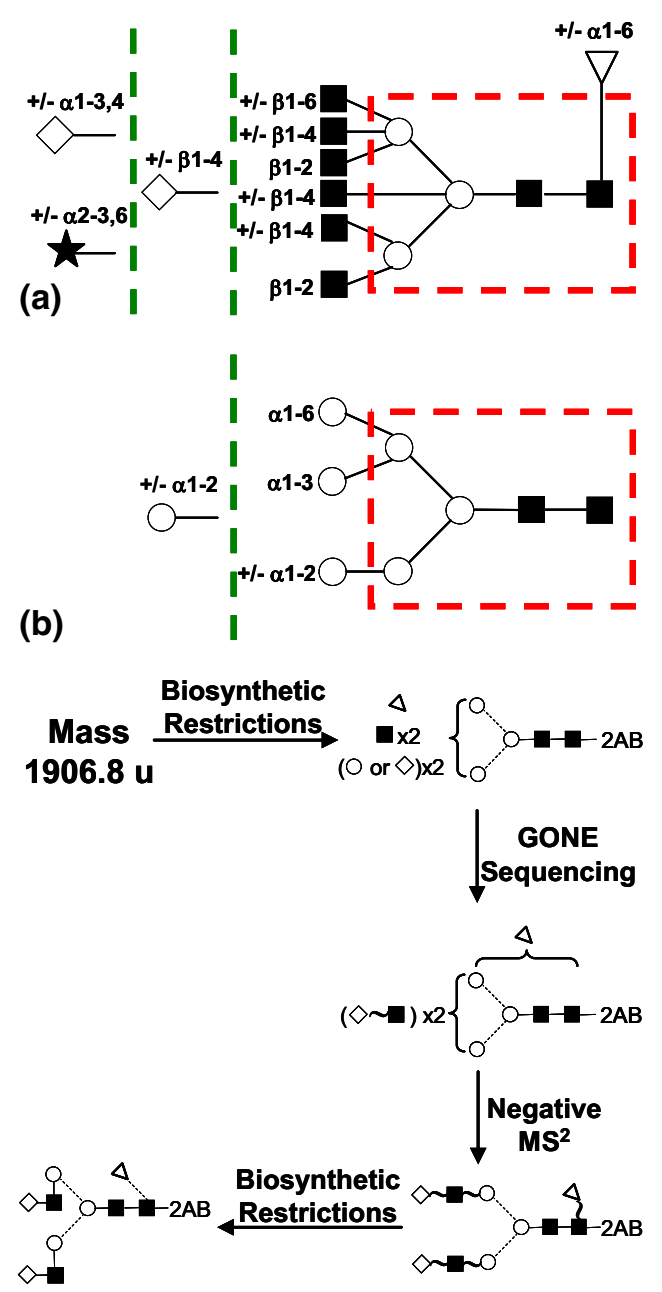

(c)

Figure 3. Possible structures for mammalian complex (a) and high mannose (b) oligosaccharides. Mammalian hybrid oligosaccharides contain a high-mannose-like 6-antenna and a complexlike 3-antenna. Data analysis of an example glycan (F4 in Figure 2) is shown in (c). Symbols for sugar residues are same as in Figure 2. Sugar residues that can be either present or absent are indicated by \pm . The connecting lines in (a) and (b) do not indicate positional linkages, whereas those in (c) are same as in Figure 2.

GlcNAc (Gal-GlcNAc, $365 \mathrm{u}$ ), as will be discussed in a later section. The $\mathrm{Y}$ ion at $\mathrm{m} / \mathrm{z} 759$ is then subject to the next round of fragmentation, resulting in a dominant ion at $\mathrm{m} / \mathrm{z} 678$ in $\mathrm{MS}^{3}$, due to the loss of another nonreducing end Man. The GONE sequencing was carried through $\mathrm{MS}^{8}$, and the identity of the sugar residue at each sequencing step was determined from the mass difference between the most abundant ions from two consecutive $\mathrm{MS}^{n}$ stages. The mass loss from the parent ions and their corresponding sugar residue(s) from the nonreducing end are summarized in Table 1. The GONE sequencing spectra for the three Man7 isomers are virtually identical, all illustrating a series loss of Man residues from the nonreducing end as shown in Supplemental Figure S1. Although GONE sequencing provides a simple sequence readout of the oligosaccharide chain from the nonreducing end to the chitobiose core, it does not reveal the composition of each antenna. Consequently, Man7 isomer structural differences can not be obtained solely from the GONE sequencing data.

To differentiate the structural isomers, $\mathrm{MS}^{2}$ spectra in the negative ion mode were used. These spectra are shown in Figure 4 for the doubly charged parent ions, and in Supplemental Figure S2 for the singly charged parent ions. The diagnostic ions for structural determination including reducing end fucosylation, terminal sugar residues, and antenna composition are summarized in Table 2. As shown in Figure 4, all M7 structural isomers yield a $Z_{R}: Y_{R}$ ion pair resulting from the cleavage of the glycosidic bond between the chitobiose core GlcNAc residues. The $\mathrm{m} / \mathrm{z}$ values of this ion pair can be used to indicate the absence $(322,340)$ or presence $(468,486)$ of core fucose on the reducing end GlcNAc. The presence of a terminal mannose residue indicated by GONE sequencing is confirmed by a $Z_{N R}: Y_{N R}$ ion pair resulting from the loss of the terminal mannose $(\mathrm{m} / \mathrm{z} 748$ and 757 respectively for M7). The compositions of the 6-antenna are revealed by a series of ions including a pair of D and D-18 ions from the concurrent cleavages of the glycosidic bonds between the $\beta 1-4$ core mannose and the core GlcNAc and that between the $\beta 1-4$ core mannose and the $\alpha 1-3$ core mannose, a pair of $C$ and $B$ ions of the 6-antenna together with the $\alpha 1-6$ core mannose $\left({ }_{6} \mathrm{C}\right.$ and $\left.{ }_{6} \mathrm{~B}\right)$, and a pair of $A$ type ions $\left({ }^{0,3} A_{R-2}\right.$ and $\left.{ }^{0,4} A_{R-2}\right)$ from cross-ring cleavage on the branching mannose. Based on this series of ions, it can be concluded that the outermost mannose is on the 6-antenna for M7-I and M7-III, and on the 3-antenna for M7-II. The M7-I and M7-III isomers can be distinguished by the ion pair of $\mathrm{D}^{\prime}$ and $\mathrm{D}^{\prime}-18$. The presence of the $m / z 485$ and 467 ion pair indicates that the $\alpha 1-2$ mannose on the 6-antenna is on the outer antenna (Figure 4a, inset). This information is used to differentiate M7-I and M7-III. It is noteworthy that the structural diagnostic ions need to be assigned as groups rather than individually, as different glycosidic or combinations of glycosidic cleavages from multiple glycan structures can result in fragment ions of identical mass. A single fragment ion, when isolated from the rest of the spectrum, is often not sufficient to indicate a specific structure feature for oligosaccharides. For example, the fragment ion at $m / z 647$ in Figure 4 can be either a B ion for M7-I and M7-III or a D ion for M7-II. It was only when all six ions in this group were considered that one could distinguish M7-II from M7-I and III. Using the approach outlined above, the structures of M5, M9, and the isoforms of M6 and M8 were also determined (Figure 2d). Whereas the sequence and antenna compositions were derived from the MS data, the exact linkages and anomeric configurations shown in Figure $2 \mathrm{~d}$ were determined from biosynthetic restrictions as illustrated in Figure 3. For example, for high mannose structures containing more than five mannose residues, the additional mannoses are always $\alpha 1-2$ linked to the inner mannose residues. Although the structures of 


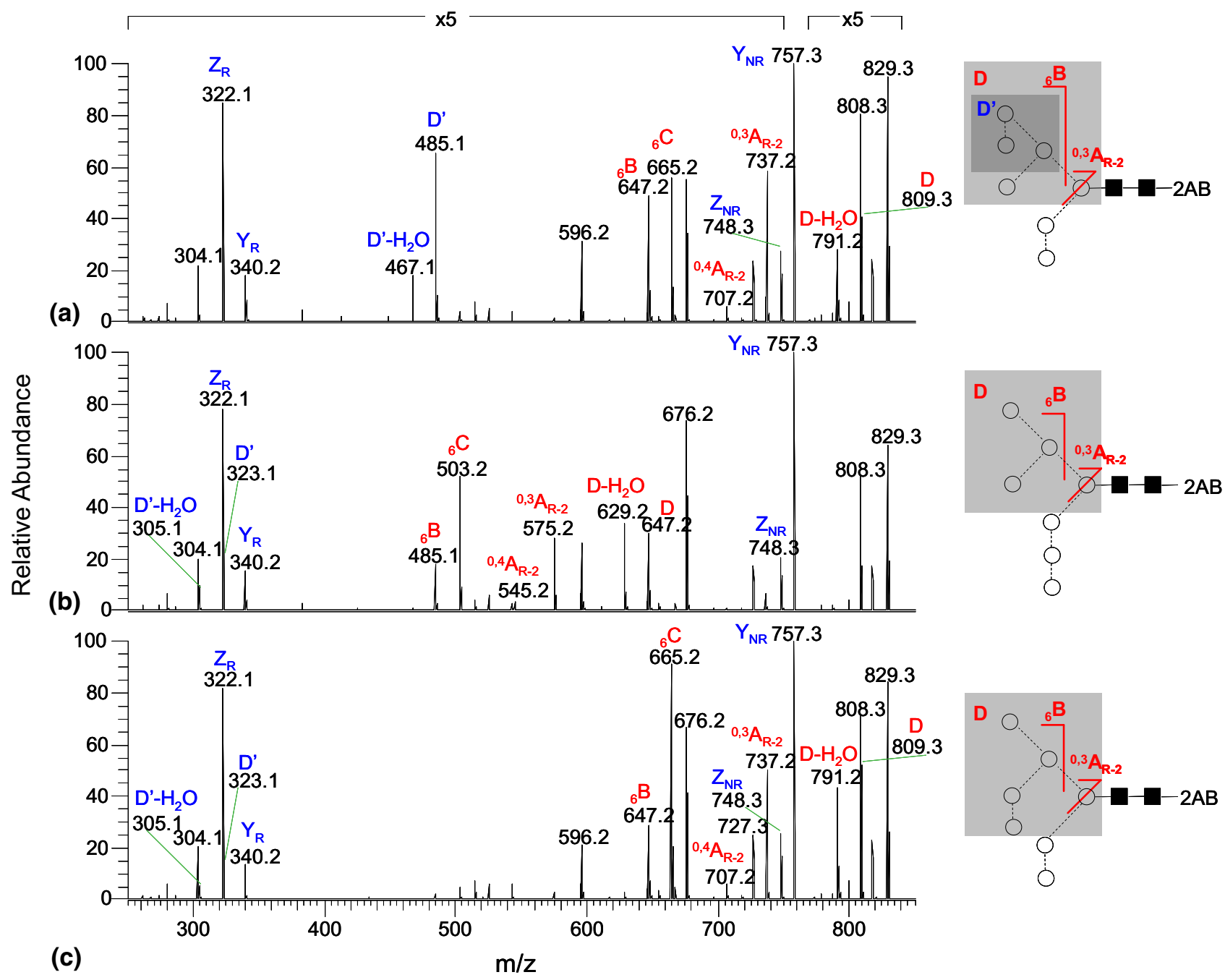

Figure 4. Negative $\mathrm{MS}^{2}$ spectra of $[\mathrm{M}-2 \mathrm{H}]^{2-}$ of M7 isomers: (a) M7-I; (b) M7-II; (c) M7-III. Ion nomenclature of Domon and Costello [53] and annotations defined in Table 2 are used in this figure and subsequent figures. Labels in red denote fragment ions indicating the 6-antenna composition. The ion pair of $Z_{R}$ and $Y_{R}\left(Z_{R}: Y_{R}\right)$ are used to determine the presence or absence of reducing end fucose. The ion pair of $Z_{N R}$ and $Y_{N R}\left(Z_{N R}: Y_{N R}\right)$ are used to determine the nonreducing end monosaccharide. The relative ion intensities in certain $\mathrm{m} / \mathrm{z}$ ranges are amplified so low abundant fragment ions can be better seen. The amplification factor are indicated as " $\mathrm{xN}^{\mathrm{N}}$ " on top of the spectra in this figure $(n=5)$ and subsequent figures. The grey boxes indicate the composition of $\mathrm{D}$ and $\mathrm{D}^{\prime}$ ions.

most high mannose glycans determined here, including the isoforms of Man7 and Man8, are consistent with previous NMR results [2], the presence of a second Man6 isoform (M6-II) was not reported in the NMR study. Since this isoform accounts for only $5 \%$ of total Man6 and $1.5 \%$ of total glycan pool released from RNase B (based on integrated fluorescence peak area in Figure 2), it is not surprising that this minor species was not confidently assigned in the NMR study, and was only revealed with the high-resolution RP separation and high sensitivity MS detection as employed here.

It is worth pointing out that detailed structural characterization by MS can be facilitated by advancement in mass spectrometers, as well as the instrument controlling software. Mass spectrometers with high- resolution can distinguish ionic species of different atomic compositions but with identical nominal mass and thus provide additional structural information. For example, the presence of the $\mathrm{D}^{\prime}$ ion at $m / z 323$ for M7-II and M7-III can be ambiguous when using a typical ion trap instrument, as this ion has the same nominal mass as the second isotopic peak of the $Z_{R}$ ion. Analysis using a high-resolution instrument, such as the Thermo LTQ Orbitrap mass spectrometer, confirmed the presence of $\mathrm{D}^{\prime}$ and $\mathrm{D}^{\prime}-18$ for M7-II and M7-III, where distinct ion signals were readily observed for $\mathrm{D}^{\prime}(\mathrm{m} / \mathrm{z} 323.10)$ and the second isotopic peak of the $Z_{R}$ ion $(m / z$ 323.14). In addition, sophisticated instrument software, such as the dynamic exclusion function of the ion trap instrument used here, enables the analyses of coeluting ionic species in a single experiment. In the case of M7 character- 
ization, singly and doubly charged parent ions of the same species were fragmented individually, adding further confidence in structural assignment. Simpler fragmentation patterns were observed for singly charged parent ions of M7 isomers, as shown in Supplemental Figure S2. In addition to the ions discussed above, a pair of $\mathrm{Z}$ and $\mathrm{Y}$ ions from the cleavage between the $\alpha 1-6$ and $\beta 1-4$ core mannoses was also evident, supporting the structural assignment.

Although as little as $70 \mathrm{fmol}$ oligosaccharide can be readily detected by this method [10], the quality of the characterization data will be dependent on the quantity of the oligosaccharide analyzed. The least abundant peak characterized here (peak R4) corresponded to oligosaccharide released from $\sim 4.5 \mathrm{pmol}$ protein, for which high quality sequence and structural information were obtained.

\section{Complex Glycans}

A variety of $\mathrm{N}$-linked complex type oligosaccharides can be synthesized in mammalian systems, which can differ in the composition of 6- and 3-antennae, the attachment site of fucose or sialic acid, and in whether they contain bisecting GlcNAc or $\alpha \mathrm{Gal}$ (Figure 3a). Similar to the approach described above for high mannose glycans, structure elucidation of complex glycans can also be achieved using the combination of GONE sequencing and negative $\mathrm{MS}^{2}$ data, together with the application of biosynthetic restriction. Briefly, in positive ion mode, GONE sequencing data not only provide compositional sequences, but also differentiate stereoisomers such as Gal and Man, and indicate the presence of bisecting GlcNAc or $\alpha \mathrm{Gal}$, as summarized in Table 1. In negative ion mode, a 6-ion series can be used to determine 6-antenna composition (and thus the complementary 3-antenna composition), as summarized in Table 2. Other structure features can also be determined from their respective diagnostic ions, such as the identity of nonreducing end terminal residue(s) $\left(Z_{\mathrm{NR}}: \mathrm{Y}_{\mathrm{NR}}\right)$, presence of fucosylation $\left(Z_{\mathrm{F}}\right)$, and whether the fucose is attached to the reducing end GlcNAc or not $\left(Z_{R}: Y_{R}\right)$ (see Table 2). To demonstrate the broad applicability of the combined approach of GONE sequencing with negative $\mathrm{MS}^{2}$ analysis, three examples of complex glycans are provided, which differ in a number of the structural features described.

\section{Tetra-Antennary with Bisecting GlcNAc}

In multi-antennary mammalian complex glycans (Figure 3a), the 6-antennae can be attached to the 2, 4, or 6 positions of the $\alpha 1-6$ core mannose, and the 3 -antennae can be attached to the 2 or 4 position of the $\alpha 1-3$ core mannose [5]. In addition, a bisecting GlcNAc can be $\beta 1-4$ linked to the $\beta 1-4$ core mannose. Detailed characterization of this type of glycan thus requires the determination of the 6- and 3-antennae compositions and identification of the bisecting GlcNAc.
Similar to that for high mannose structures, GONE sequencing experiments of complex oligosaccharides provided straightforward readouts. The peak O2 (Figure 2 b) from a commercial ovalbumin sample is provided as an example. The mass of this $2 \mathrm{AB}$ labeled glycan indicated a $(\mathrm{GlcNAc})_{5}$ composition in addition to the trimannosyl chitobiose core structure. GONE sequencing spectra are shown in Figure 5. The mass difference of the most abundant ions in consecutive $\mathrm{MS}^{n}$ spectra was calculated to determine the sugar residue released from each sequencing step. In the $\mathrm{MS}^{2}$ and $\mathrm{MS}^{3}$ steps, loss of nonreducing end GlcNAc was accompanied by the loss of a charging proton, as indicated in Figure 5. Whereas the loss of GlcNAc is evident from $\mathrm{MS}^{2}$ to $\mathrm{MS}^{5}$, the fifth GlcNAc was not released until both core $\alpha$-mannose residues were lost $\left(\mathrm{MS}^{8}\right.$, bottom spectrum in Figure 5). This last GlcNAc (not counting those of the chitobiose core) is a bisecting GlcNAc, which is confirmed by negative $\mathrm{MS}^{2}$ analysis as will be discussed later. Analysis of many other oligosaccharides containing bisecting GlcNAc confirmed that the bisecting GlcNAc is always released at later stages of tandem MS, more specifically, after the loss of one or both core $\alpha$-mannoses (see, for example, Supplemental Figure S6). This allows straightforward identification of bisecting GlcNAc, which is difficult to identify using exclusively an exoglycosidase digestion approach. The attachment sites of the four early released GlcNAc (i.e., the composition of 6- and 3-antennae), can not be determined by GONE sequencing data alone. This information, however, can be obtained from the negative $\mathrm{MS}^{2}$ spectrum.

Peak O2 and peak O3 (Figure 2b) contained ionic species of the same mass. GONE sequencing data were

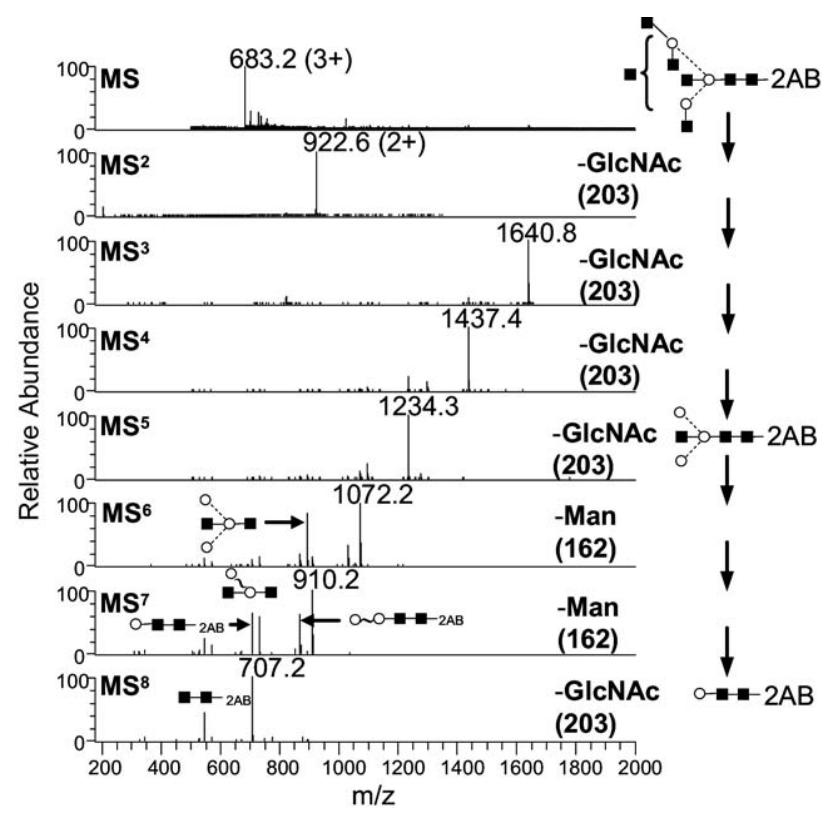

Figure 5. GONE sequencing spectra for glycan O2. Sequential losses of sugar residues from the nonreducing end after each sequencing step are indicated with their corresponding mass loss in parentheses. 


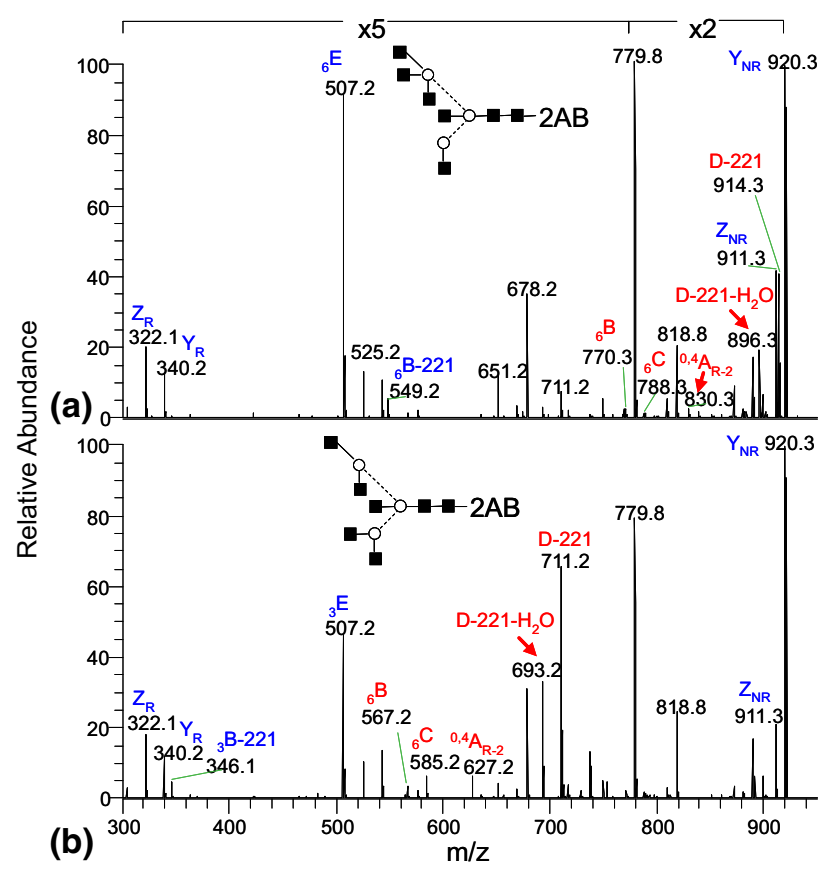

Figure 6. Negative $\mathrm{MS}^{2}$ spectra of $[\mathrm{M}-2 \mathrm{H}]^{2-}$ of: (a) glycan $\mathrm{O} 2$; (b) glycan $\mathrm{O}$.

very similar for these two oligosaccharide species, both of which indicated a bisecting GlcNAc. Structural differences between the two species were revealed in their $\mathrm{MS}^{2}$ spectra under the negative ion mode, as indicated in Figure 6. The $Z_{\mathrm{R}}: Y_{\mathrm{R}}$ ion pair at $\mathrm{m} / \mathrm{z} 322$ and 340 confirmed the lack of core fucosylation on the reducing end GlcNAc. Only one $Z_{\mathrm{NR}}: Y_{\mathrm{NR}}$ ion pair at $\mathrm{m} / \mathrm{z} 911$ and 920 was observed, indicating that GlcNAc is the only nonreducing end residue for both structures. Instead of the ion pair of D and D-18, oligosaccharides with bisecting GlcNAc produce D-221 and D-221- $\mathrm{H}_{2} \mathrm{O}$ ions (see Table 2), revealing the 6-antenna composition. ${ }^{0,3} \mathrm{~A}_{\mathrm{R}-2}$ is missing in these spectra, but ${ }^{0,4} \mathrm{~A}_{\mathrm{R}-2}$ and ${ }_{6} \mathrm{~B},{ }_{6} \mathrm{C}$ ion pair can still be seen. Based on the $m / z$ values of this ion series, the $\mathrm{O} 2$ oligosaccharide was determined to have three GlcNAc on the 6-antenna and only one on the 3-antenna. In contrast, the oligosaccharide under peak O3 has two GlcNAc residues on both antennae. B-221 type ions were also observed for both $\mathrm{O} 2$ and $\mathrm{O} 3$ oligosaccharides. The B-221 type ions have not been reported before. Similar to the D-221 signature ion of a bisecting GlcNAc (1-4 linked to the $\beta 1-4$ core mannose), the B-221 ion can be used to detect 1-4 linked GlcNAc on the antenna. The B-221 ion at $m / z 549$ for $\mathrm{O} 2$ indicates an antenna of three GlcNAc residues with one 1-4 linked GlcNAc. The B-221 ion at $m / z 346$ for O3 indicates an antenna of two GlcNAc residues, one of which is 1-4 linked. The biosynthetic restrictions (Figure 3) were then applied to determine the remaining linkages as shown in Figure 6.

\section{Fucosylated Bi-Antennary with or without $\alpha \mathrm{Gal}$}

Complete complex oligosaccharide structural assignment also requires determination of the fucose attach- ment site and differentiation of monosaccharide isomers. In mammalian complex glycans, fucose can be $\alpha$-linked to either the antennae or the reducing end GlcNAc. In invertebrates, the fucose attached to the core can be attached to either of the two GlcNAc residues [55]. When characterizing oligosaccharides using MS approaches, an additional challenge comes from the differentiation of monosaccharides of identical mass. However, identification of stereoisomers such as Man and Gal, and anomers such as $\beta \mathrm{Gal}$ and $\alpha \mathrm{Gal}$, are an expected part of a thorough primary glycan structural determination. Such information can be derived from a combination of GONE sequencing and negative $\mathrm{MS}^{2}$ analysis. In the following two examples (both fucosylated bi-antennary complex glycans released from a commercial fetuin sample), GONE sequencing with negative $\mathrm{MS}^{2}$ have been used to identify glycan primary structures.

Taking into account the biosynthetic restrictions, the masses of peak F3 and F4 (Figure 2c) indicate oligosaccharide compositions of $(\mathrm{Hex})_{7}(\mathrm{GlcNAc})_{4}(\mathrm{Fuc})$ and $(\mathrm{Hex})_{5}(\mathrm{GlcNAc})_{4}(\mathrm{Fuc})$, respectively. Tabular summaries of the GONE sequencing data for both structures are shown in Supplemental Figure S3. In GONE sequencing, each sequencing step does not always release a single sugar residue. As indicated in Table 1, di- or trisaccharides of specific sequences can also be released in a single sequencing step. For peak F4, the first round of GONE sequencing revealed a nonreducing end GalGlcNAc group with a mass loss of $365 \mathrm{u}$. Analysis of other glycan standards (data not shown) indicated that Gal residues, in contrast to mannose residues, are always lost together with the attaching GlcNAc. This observation is consistent with previously reported findings under similar conditions [10, 11]. This property unambiguously differentiates Gal from Man in GONE sequencing even though these monosaccharides have the same mass. It is also evident from the GONE sequencing data in Supplemental Figure S3a (table) that the fucose residue is attached to the core structure, since it was released after both Gal-GlcNAc antennae. The GONE sequencing data for peak F3 is shown in Supplemental Figure S3b (table). In the first two sequencing steps, a mass loss of $527 \mathrm{u}$ was observed, corresponding to a $(\mathrm{Hex})_{2}$ GlcNAc group. Similar to the discussion above, this mass loss can not be explained by GalGlcNAc-Man, as the Gal-GlcNAc disaccharide and Man residue would not be released in a single sequencing step. As a result, the mass loss of $527 \mathrm{u}$ in a GONE sequencing step can only be explained by a Gal-GalGlcNAc sequence for mammalian glycans (see Figure 3a). The nonreducing end Gal in the Gal-Gal-GlcNAc sequence is most likely $\alpha$ linked [56,57] as a terminal $\operatorname{Gal}(\beta 1-4)-G a l(\beta 1-4)-G l c N A c$ sequence in a N-linked glycan has only been found in fish [58] but not in mammals. Just as the mass loss of $365 \mathrm{u}$ is diagnostic of a Gal-GlcNAc group, the mass loss of $527 \mathrm{u}$ in GONE sequencing can be used as an indication of nonreducing end $\alpha$ Gal-Gal-GlcNAc for mammalian glycans. Similar 
to glycan F4, glycan F3 GONE sequencing data revealed a core fucose (Supplemental Figure S3b table). For both structures, however, the GONE sequencing data cannot define which GlcNAc is attached to this core fucose.

In the negative ion mode $\mathrm{MS}^{2}$ spectra, the most abundant ion for both structures is a $\mathrm{Z}$ type ion, resulting from the loss of the fucose residue $\left(Z_{F}\right.$, Supplemental Figure S3a and S3b). The $Z_{R}: Y_{R}$ ion pair at $\mathrm{m} / \mathrm{z} 468$ and 486 indicates that the core fucose is attached to the reducing end GlcNAc. Since the GONE sequencing data clearly indicated the presence of a core fucose, if the $Z_{R}: Y_{R}$ ion pair were to be at $m / z 322$ and 340 , those values would indicate a fucose attached to the second GlcNAc from the reducing end. The linkage position on the sugar ( $\alpha 1-3$ for plants, $\alpha 1-6$ for mammals, or both for invertebrates [55]), could not be determined from these data. For glycan F4, $\mathrm{Z}_{\mathrm{NR}}: \mathrm{Y}_{\mathrm{NR}}$ ion pair at $\mathrm{m} / \mathrm{z} 862$ and 871 confirms the presence of terminal Gal. Interestingly, an abundant ion pair at $\mathrm{m} / \mathrm{z}$ 943 and 952 was observed for F3, resulting from the loss of Gal-Gal (i.e., $\mathrm{Z}_{\mathrm{NR}-1}: \mathrm{Y}_{\mathrm{NR}-1}$ ). Thus, an abundant ion pair at mass loss of 342 and $324 \mathrm{u}$ (as opposed to the mass loss of 180 and $162 \mathrm{u}$ ) indicates terminal $\alpha \mathrm{Gal}-\mathrm{Gal}$. Other $\mathrm{F}$ type ion $\mathrm{m} / \mathrm{z}$ value differences, 424 for $\mathrm{F} 4$ and 586 for F3, indicate terminal Gal-GlcNAc and Gal-GalGlcNAc, respectively, as described by Harvey et al. [32] The 6-antenna composition of both oligosaccharide species can be derived from the ion series of $\mathrm{D}, \mathrm{D}-\mathrm{H}_{2} \mathrm{O}$, ${ }^{0,3} \mathrm{~A}_{\mathrm{R}-2,}{ }^{0,4} \mathrm{~A}_{\mathrm{R}-2},{ }_{6} \mathrm{~B}$, and ${ }_{6} \mathrm{C}$, in a manner similar to the oligosaccharide structures discussed in the previous sections. It is worth pointing out that each of F3 and F4 glycans comprise only a very small fraction of the total glycan pool released from the commercial fetuin sample ( $2 \%$ or less based on integrated fluorescence peak area). Whereas sialylated fetuin glycans have been studied in great detail by NMR [3], little was known for low abundant neutral glycans in fetuin. It can not be ruled out that some of the minor glycan species identified in this study might originate from other glycoprotein contaminants since the glycoproteins obtained from commercial sources were analyzed directly without further purification. This possibility was illustrated for a commercial ovalbumin sample [54]. Regardless, this study demonstrates that low abundant glycan species that could be difficult to characterize using traditional methods can be readily identified using the LC/MS approach described here.

\section{Sialylated Complex}

Sialic acid is commonly the terminal sugar in mammalian glycans, linked to a Gal residue. The sialic acid attachment site can sometimes be determined through GONE sequencing. Sialic acid tends to be cleaved off together with the Gal-GlcNAc to which it is attached. This provides a convenient way to determine the location of the sialic acid, exemplified in the GONE sequencing of oligosaccharide F2 (Figure 2c). GONE sequencing data for this sialylated glycan is shown in
Supplemental Figure S4 (table). The first round of sequencing indicated a Gal-Gal-GlcNAc sequence at the nonreducing end, as evidenced by its characteristic ion loss of $527 \mathrm{u}$. The second round yielded a mass loss of $657 \mathrm{u}$, resulting from the loss of SA-Gal-GlcNAc. These results strongly suggest that the sialic acid is attached to Gal-GlcNAc, not the $\alpha \mathrm{Gal}$ in the Gal-Gal-GlcNAc group. Further sequencing rounds indicated the loss of Man and Fuc from the fucosylated core structure. Negative $\mathrm{MS}^{2}$ analysis was then used to determine the location of the SA-Gal-GlcNAc and Gal-Gal-GlcNAc antennae.

In the negative $\mathrm{MS}^{2}$ spectrum shown in Supplemental Figure S4, the $Z_{R}: Y_{R}$ ion pair at $m / z 468$ and 486 confirmed that the fucose was attached to the reducing end GlcNAc, and the $Z_{\mathrm{NR}-1}: Y_{\mathrm{NR}-1}$ ion pair at $m / z 1008$ and 1017 (mass loss of 342 and 324) confirmed the presence of an $\alpha \mathrm{Gal}-\mathrm{Gal}$ group. A $\mathrm{Z}_{\mathrm{NR}}: \mathrm{Y}_{\mathrm{NR}}$ ion pair corresponding to the loss of SA was not observed. Since the doubly charged parent ion would likely lose a charge with SA loss, the resulting singly charged ion would have an $m / z$ value outside that available in this analysis. The 6-antenna diagnostic ion series, including the $\mathrm{D}$ ion at $m / z$ 850, indicated that the Gal-Gal-GlcNAc group is attached to the 6-antenna, and thus the SA-Gal-GlcNAc group must be attached to the 3-antenna. The presence of the Gal-Gal-GlcNAc sequence is also supported by the $\mathrm{F}$ ion at $m / z 586$ and the $C$ and $B$ ion pair at $m / z 544$ and 526. The presence of SA-Gal-GlcNAc is supported by the two B ions at $m / z 655$ and 817 . However, the exact linkage of SA to Gal ( $\alpha 2-3$ or $\alpha 2-6)$, can not be determined from this analysis.

\section{Hybrid Glycans}

The diagnostic ions derived from GONE sequencing and negative $\mathrm{MS}^{2}$ spectra of the high mannose and complex oligosaccharides discussed above (Table 1 and Table 2) can be readily applied to many other oligosaccharide structures. Two hybrid oligosaccharides will be discussed as examples to illustrate the utility of this technique.

\section{Sialylated Hybrid}

GONE sequencing of oligosaccharide F1 (Figure 2c) revealed the nonreducing ends to be the SA-Gal-GlcNAc sequence and a Man residue, as shown in Supplemental Figure S5 (table). The $\alpha 1-6$ core Man has two additional Man residues attached to it, as elucidated by the 6antenna diagnostic ion series with $\mathrm{D}$ ion at $\mathrm{m} / \mathrm{z} 647$. The SA-Gal-GlcNAc is thus attached to the 3-antenna, with ${ }_{3} \mathrm{~B}$ and ${ }_{3} \mathrm{~B}_{3}$ at 817 and 655 , respectively. In addition to the $Z_{N R}: Y_{N R}$ ion pair at $m / z 914$ and 923 , indicating the presence of terminal Man, another $\mathrm{Y}_{\mathrm{NR}}$ ion is also observed at $m / z 1719$ as a singly charged ion (not shown). The latter $Y_{N R}$ ion indicates the presence of terminal SA, and also confirmed that one negative charge is located on the SA residue. 


\section{Hybrid with Bisecting GlcNAc}

The GONE sequencing data of oligosaccharide O1 (Figure $2 b$ ) revealed the nonreducing end GlcNAc and Man residues, as shown in Supplemental Figure S6 (table). The GlcNAc released at MS ${ }^{7}$ indicates that this GlcNAc is $\beta 1-4$ linked to the $\beta 1-4$ core mannose as a bisecting GlcNAc. This is confirmed by the presence of D-221 instead of a D ion in the negative $\mathrm{MS}^{2}$ spectrum. The 6-antenna diagnostic ion series indicates the attachment of two Man residues on the $\alpha 1-6$ core Man. Thus, the nonreducing end GlcNAc is attached to the 3-antenna, consistent with the $\mathrm{F}$ ion at $m / z$ 262. The two $\mathrm{Z}_{\mathrm{NR}}: \mathrm{Y}_{\mathrm{NR}}$ ion pairs confirmed that both terminal Man and terminal GlcNAc are present.

\section{Conclusions}

An online RP-HPLC $/ \mathrm{MS}^{n}$ method described here was used for rapid structural analysis of mammalian Nlinked oligosaccharides. Complementary information was obtained by combining MS data acquired in both positive and negative ion modes through polarity switching within a single chromatographic analysis.

In the positive ion mode, single sugar or multiple sugars are released in each round of $\mathrm{MS}^{n}$. These GONE sequencing data provide a simple readout of the glycan sequence, similar to that of the classic exoglycosidase sequencing approach. Although lacking the linkage and anomeric specificities in glycosidase digestions, GONE sequencing provides much higher speed and sensitivity, and is not limited by availability of specific enzymes. Since only one prominent $\mathrm{Y}$ ion is generated for a wide variety of glycans under the GONE sequencing condition, the selection of the parent ion at each sequencing step is straightforward. As many as eight sequencing cycles can be automatically programmed within a $15 \mathrm{~s}$ positive mode cycle switch, sufficient to thoroughly sequence all but the most complex glycans. Identities of sugar residues from the nonreducing end can be easily determined from the mass differences of the most abundant ions in consecutive $\mathrm{MS}^{n}$ spectra. Stereoisomers such as Gal and Man can also be distinguished based on their different fragmentation properties. Some structural features can be revealed from the GONE sequencing experiment as well, such as the attachment site of fucose and SA, and the presence of bisecting GlcNAc and $\alpha \mathrm{Gal}$. The presence of bisecting GlcNAc, a structural feature that can be difficult to obtain using the classic exoglycosidase sequencing approach, is readily revealed simply based on the readout order in GONE sequencing. Due to the branched structures of oligosaccharides, a linear sequence readout as in peptide/protein sequencing does not apply for multiple antennary glycans. Similar to the classic exoglycosidase sequencing technique, the exact branch location of the sugar residue(s) released at each sequencing step can not be determined in GONE se- quencing. Such information, however, can be derived from MS $^{2}$ analysis in negative ion mode.

In the negative ion mode, diagnostic ions similar to those observed under higher collision energies are also observed using an ion trap instrument with collision energy of only a couple $\mathrm{eV}$. The composition of the antennae can be readily derived from the 6-antenna diagnostic ion series. The interpretation of the negative $\mathrm{MS}^{2}$ data is greatly simplified with the additional restrictions from GONE sequencing results, where the terminal sugars or sugar groups of specific sequences were revealed and used to reduce the number of possible antenna compositions to be considered. The $\mathrm{Z}_{\mathrm{R}}: \mathrm{Y}_{\mathrm{R}}$ ion pair can be used to determine the location of the core fucose, and the identity of the terminal residue (or the terminal group in the case of $\alpha \mathrm{Gal}-\mathrm{Gal}$ sequence) is revealed by the ion pair of $Z_{N R(-1)}$ and $Y_{N R(-1)}$. The structure of some subunits can also be determined or confirmed by other diagnostic ions. This information, together with the terminal sequence revealed by GONE sequencing, can be used to determine the structures of even very complex glycans.

Although other techniques may still be required for the determination of certain linkages (such as $\alpha 1-3,6$ Fuc and $\alpha 2-3,6 \mathrm{SA}$ ) and structures of aberrant glycans deviating from normal biosynthetic restrictions, the GONE sequencing method, in combination with the supplemental information from negative $\mathrm{MS}^{2}$ analysis, provides a detailed picture of the oligosaccharide structure. This technique is thus a very useful tool for detailed characterization of oligosaccharides with little sample consumption and high speed, and can be readily applied to structural elucidation of unknown glycan species. When combined with high-resolution RP-HPLC analysis [10], both structural elucidation and quantification of even minor glycan species in a highly complex mixture can be obtained within a single chromatographic run.

\section{Acknowledgments}

The authors thank G. Rogers, Z. Zhang, I. Apostol, R. Remmele, and D. Kelner for critical review of the manuscript.

\section{Appendix A Supplementary Material}

Supplementary material associated with this article may be found in the online version at doi:10.1016/ j.jasms.2009.06.003.

\section{References}

1. Rademacher, T. W.; Parekh, R. B.; Dwek, R. A. Glycobiol. Annu. Rev. Biochem. 1988, 57, 785-838.

2. Fu, D.; Chen, L.; O'Neill, R. A. A detailed structural characterization of ribonuclease B oligosaccharides by $1 \mathrm{H}$ NMR spectroscopy and mass spectrometry. Carbohydr. Res. 1994, 261, 173-186.

3. Green, E. D.; Adelt, G.; Baenziger, J. U.; Wilson, S.; Van Halbeek, H. The asparagine-linked oligosaccharides on bovine fetuin. Structural analysis of N-glycanase-released oligosaccharides by $500-\mathrm{MHz} 1 \mathrm{H}$ NMR spectroscopy. J. Biol. Chem. 1988, 263, 18253-18268. 
4. Takahashi, N.; Ishii, I.; Ishihara, H.; Mori, M.; Tejima, S.; Jefferis, R.; Endo, S.; Arata, Y. Comparative structural study of the N-linked oligosaccharides of human normal and pathological immunoglobulin G. Biochemistry 1987, 26, 1137-1144.

5. Yamashita, K.; Kamerling, J. P.; Kobata, A. Structural study of the carbohydrate moiety of hen ovomucoid. Occurrence of a series of penta-antennary complex-type asparagine-linked sugar chains. J. Biol. Chem. 1982, 257, 12809-12814

6. Callewaert, N.; Geysens, S.; Molemans, F.; Contreras, R. Ultrasensitive profiling and sequencing of N-linked oligosaccharides using standard DNA-sequencing equipment. Glycobiology 2001, 11, 275-281.

7. Morelle, W.; Michalski, J. C. The mass spectrometric analysis of glycoproteins and their glycan structures. Curr. Anal. Chem. 2005, 1, 29-57

8. Rudd, P. M.; Guile, G. R.; Kuster, B.; Harvey, D. J.; Opdenakker, G.; Dwek, R. A. Oligosaccharide sequencing technology. Nature 1997, 388, 205-207.

9. Zaia, J. Mass spectrometry of oligosaccharides. Mass Spectrom. Rev. 2004, 23, 161-227.

10. Chen, X.; Flynn, G. C. Analysis of N-glycans from recombinant immunoglobulin $\mathrm{G}$ by on-line reversed-phase high-performance liquid chromatography/mass spectrometry. Anal. Biochem. 2007, 370, 147-161.

11. Ojima, N.; Masuda, K.; Tanaka, K.; Nishimura, O. Analysis of neutral oligosaccharides for structural characterization by matrix-assisted laser desorption/ionization quadrupole ion trap time-of-flight mass spectrometry. J. Mass Spectrom. 2005, 40, 380-388.

12. Sato, Y.; Suzuki, M.; Nirasawa, T.; Suzuki, A.; Endo, T. Microsequencing of glycans using 2-aminobenzamide and MALDI-TOF mass spectrometry: Occurrence of unique linkage-dependent fragmentation. Anal. Chem. 2000, 72, 1207-1216.

13. Ashline, D. J.; Lapadula, A. J.; Liu, Y. H.; Lin, M.; Grace, M.; Pramanik, B.; Reinhold, V. N. Carbohydrate structural isomers analyzed by sequential mass spectrometry. Anal. Chem. 2007, 79, 3830-3842.

14. Harvey, D. J. Electrospray mass spectrometry and fragmentation of $\mathrm{N}$-linked carbohydrates derivatized at the reducing terminus. J. Am. Soc. Mass Spectrom. 2000, 11, 900-915.

15. Harvey, D. J.; Bateman, R. H.; Green, M. R. High-energy collisioninduced fragmentation of complex oligosaccharides ionized by matrixassisted laser desorption/ionization mass spectrometry. J. Mass Spectrom. 1997, 32, 167-187.

16. Lemoine, J.; Fournet, B.; Despeyroux, D.; Jennings, K. R.; Rosenberg, R.; de Hoffmann, E. Collision-induced dissociation of alkali metal cationized and permethylated oligosaccharides: Influence of the collision energy and of the collision gas for the assignment of linkage position. J. Am. Soc. Mass Spectrom. 1993, 4, 197-203.

17. Lewandrowski, U.; Resemann, A.; Sickmann, A. Laser-induced dissociation/high-energy collision-induced dissociation fragmentation using MALDI-TOF/TOF-MS instrumentation for the analysis of neutral and acidic oligosaccharides. Anal. Chem. 2005, 77, 3274-3283.

18. Mechref, Y.; Kang, P.; Novotny, M. V. Differentiating structural isomers of sialylated glycans by matrix-assisted laser desorption/ionization time-of-flight/time-of-flight tandem mass spectrometry. Rapid Commun. Mass Spectrom. 2006, 20, 1381-1389.

19. Mechref, Y.; Novotny, M. V.; Krishnan, C. Structural characterization of oligosaccharides using MALDI-TOF/TOF tandem mass spectrometry. Anal. Chem. 2003, 75, 4895-4903.

20. Morelle, W.; Slomianny, M. C.; Diemer, H.; Schaeffer, C.; van Dorsselaer, A.; Michalski, J. C. Fragmentation characteristics of permethylated oligosaccharides using a matrix-assisted laser desorption/ionization two-stage time-of-flight (TOF/TOF) tandem mass spectrometer. Rapid Commun. Mass Spectrom. 2004, 18, 2637-2649.

21. Morelle, W.; Slomianny, M. C.; Diemer, H.; Schaeffer, C.; van Dorsselaer, A.; Michalski, J. C. Structural characterization of 2-aminobenzamidederivatized oligosaccharides using a matrix-assisted laser desorption/ ionization two-stage time-of-flight tandem mass spectrometer. Rapid Commun. Mass Spectrom. 2005, 19, 2075-2084.

22. Spina, E.; Sturiale, L.; Romeo, D.; Impallomeni, G.; Garozzo, D.; Waidelich, D.; Glueckmann, M. New fragmentation mechanisms in matrixassisted laser desorption/ionization time-of-flight/time-of-flight tandem mass spectrometry of carbohydrates. Rapid Commun. Mass Spectrom. 2004, 18, 392-398.

23. Stephens, E.; Maslen, S. L.; Green, L. G.; Williams, D. H. Fragmentation characteristics of neutral N-linked glycans using a MALDI-TOF/TOF tandem mass spectrometer. Anal. Chem. 2004, 76, 2343-2354.

24. Wuhrer, M.; Deelder, A. M. Negative-mode MALDI-TOF/TOF-MS of oligosaccharides labeled with 2-aminobenzamide. Anal. Chem. 2005, 77, 6954-6959.

25. Wuhrer, M.; Deelder, A. M. Matrix-assisted laser desorption/ionization in-source decay combined with tandem time-of-flight mass spectrometry of permethylated oligosaccharides: Targeted characterization of specific parts of the glycan structure. Rapid Commun. Mass Spectrom. 2006, 20, 943-951.

26. Chai, W.; Piskarev, V.; Lawson, A. M. Branching pattern and sequence analysis of underivatized oligosaccharides by combined MS/MS of singly and doubly charged molecular ions in negative-ion electrospray mass spectrometry. J. Am. Soc. Mass Spectrom. 2002, 13, $670-679$.
27. Cheng, H. L.; Pai, P. J.; Her, G. R. Linkage and branch determination of $\mathrm{N}$-linked oligosaccharides using sequential degradation/closed-ring chromophore labeling/negative ion trap mass spectrometry. J. Am. Soc. Mass Spectrom. 2007, 18, 248-259.

28. Harvey, D. J. Fragmentation of negative ions from carbohydrates: part 3. Fragmentation of hybrid and complex N-linked glycans. J. Am. Soc. Mass Spectrom. 2005, 16, 647-659.

29. Harvey, D. J. Fragmentation of negative ions from carbohydrates: Part 2. Fragmentation of high-mannose N-linked glycans. J. Am. Soc. Mass Spectrom. 2005, 16, 631-646.

30. Harvey, D. J. Fragmentation of negative ions from carbohydrates: part 1. Use of nitrate and other anionic adducts for the production of negative ion electrospray spectra from $\mathrm{N}$-linked carbohydrates. J. Am. Soc. Mass Spectrom. 2005, 16, 622-630.

31. Harvey, D. J. Collision-induced fragmentation of negative ions from $\mathrm{N}$-linked glycans derivatized with 2-aminobenzoic acid. J. Mass Spectrom. 2005, 40, 642-653.

32. Harvey, D. J.; Royle, L.; Radcliffe, C. M.; Rudd, P. M.; Dwek, R. A. Structural and quantitative analysis of N-linked glycans by matrixassisted laser desorption ionization and negative ion nanospray mass spectrometry. Anal. Biochem. 2008, 376, 44-60.

33. Pfenninger, A.; Karas, M.; Finke, B.; Stahl, B. Structural analysis of underivatized neutral human milk oligosaccharides in the negative ion mode by nano-electrospray MS $(n)$. Part 2: Application to isomeric mixtures. J. Am. Soc. Mass Spectrom. 2002, 13, 1341-1348.

34. Oian, J.; Liu, T.; Yang, L.; Daus, A.; Crowley, R.; Zhou, O. Structural characterization of N-linked oligosaccharides on monoclonal antibody cetuximab by the combination of orthogonal matrix-assisted laser desorption/ionization hybrid quadrupole-quadrupole time-of-flight tandem mass spectrometry and sequential enzymatic digestion. Anal. Biochem. 2007, 364, 8-18.

35. Sagi, D.; Peter-Katalinic, J.; Conradt, H. S.; Nimtz, M. Sequencing of tri- and tetra-antennary N-glycans containing sialic acid by negative mode ESI QTOF tandem MS. J. Am. Soc. Mass Spectrom. 2002, 13, $1138-1148$

36. Wong, A. W.; Cancilla, M. T.; Voss, L. R.; Lebrilla, C. B. Anion dopant for oligosaccharides in matrix-assisted laser desorption/ionization mass spectrometry. Anal. Chem. 1999, 71, 205-211.

37. Bruggink, C.; Wuhrer, M.; Koeleman, C. A.; Barreto, V.; Liu, Y.; Pohl, C.; Ingendoh, A.; Hokke, C. H.; Deelder, A. M. Oligosaccharide analysis by capillary-scale high-pH anion-exchange chromatography with on-line ion-trap mass spectrometry. J. Chromatogr. B 2005, 829, 136-143.

38. Cheng, H. L.; Her, G. R. Determination of linkages of linear and branched oligosaccharides using closed-ring chromophore labeling and negative ion trap mass spectrometry. J. Am. Soc. Mass Spectrom. 2002, 13, 1322-1330.

39. Delaney, J.; Vouros, P. Liquid chromatography ion trap mass spectrometric analysis of oligosaccharides using permethylated derivatives. Rapid Commun. Mass Spectrom. 2001, 15, 325-334.

40. Deguchi, K.; Takegawa, Y.; Ito, H.; Miura, N.; Yoshioka, S.; Nagai, S. Nakagawa, H.; Nishimura, S. Structural assignment of isomeric 2-aminopyridine-derivatized monosialylated biantennary N-linked oligosaccharides using negative-ion multistage tandem mass spectral matching. Rapid Commun. Mass Spectrom. 2006, 20, 412-418.

41. Takegawa, Y.; Deguchi, K.; Ito, S.; Yoshioka, S.; Nakagawa, H.; Nishimura, S. Simultaneous analysis of 2-aminopyridine-derivatized neutral and sialylated oligosaccharides from human serum in the negative-ion mode by sonic spray ionization ion trap mass spectrometry. Anal. Chem. 2005, 77, 2097-2106.

42. Takegawa, Y.; Deguchi, K.; Ito, S.; Yoshioka, S.; Nakagawa, H.; Nishimura, S. Structural assignment of isomeric 2-aminopyridine-derivatized oligosaccharides using negative-ion $\mathrm{MS}^{n}$ spectral matching. Rapid Commun. Mass Spectrom. 2005, 19, 937-946.

43. Takegawa, Y.; Deguchi, K.; Ito, S.; Yoshioka, S.; Sano, A.; Yoshinari, K.; Kobayashi, K.; Nakagawa, H.; Monde, K.; Nishimura, S. Assignment and quantification of 2-aminopyridine derivatized oligosaccharide isomers coeluted on reversed-phase HPLC/MS by $\mathrm{MS}^{n}$ spectral library. Anal. Chem. 2004, 76, 7294-7303.

44. Takegawa, Y.; Deguchi, K.; Nakagawa, H.; Nishimura, S. Structural analysis of an N-glycan with " $\beta 1-4$ bisecting branch" from human serum IgG by negative-ion $\mathrm{MS}^{n}$ spectral matching and exoglycosidase digestion. Anal. Chem. 2005, 77, 6062-6068.

45. Takegawa, Y.; Ito, S.; Yoshioka, S.; Deguchi, K.; Nakagawa, H.; Monde, K.; Nishimura, S. Structural assignment of isomeric 2-aminopyridinederivatized oligosaccharides using $\mathrm{MS}^{n}$ spectral matching. Rapid Commun. Mass Spectrom. 2004, 18, 385-391.

46. Costello, C. E.; Contado-Miller, J. M.; Cipollo, J. F. A glycomics platform for the analysis of permethylated oligosaccharide alditols. J. Am. Soc. Mass Spectrom. 2007, 18, 1799-1812.

47. Sheeley, D. M.; Reinhold, V. N. Structural characterization of carbohydrate sequence, linkage, and branching in a quadrupole Ion trap mass spectrometer: neutral oligosaccharides and N-linked glycans. Anal. Chem. 1998, 70, 3053-3059.

48. Weiskopf, A. S.; Vouros, P.; Harvey, D. J. Characterization of oligosaccharide composition and structure by quadrupole ion trap mass spectrometry. Rapid Commun. Mass Spectrom. 1997, 11, 1493-1504. 
49. Weiskopf, A. S.; Vouros, P.; Harvey, D. J. Electrospray ionization-ion trap mass spectrometry for structural analysis of complex N-linked glycoprotein oligosaccharides. Anal. Chem. 1998, 70, 4441-4447.

50. Bigge, J. C.; Patel, T. P.; Bruce, J. A.; Goulding, P. N.; Charles, S. M.; Parekh, R. B. Nonselective and efficient fluorescent labeling of glycans using 2-amino benzamide and anthranilic acid. Anal. Biochem. 1995, 230, 229-238.

51. Anumula, K. R. Advances in fluorescence derivatization methods for high-performance liquid chromatographic analysis of glycoprotein carbohydrates. Anal. Biochem. 2006, 350, 1-23.

52. Morelle, W.; Page, A.; Michalski, J. C. Electrospray ionization ion trap mass spectrometry for structural characterization of oligosaccharides derivatized with 2-aminobenzamide. Rapid Commun. Mass Spectrom. 2005, 19, 1145-1158.

53. Domon, B.; Costello, C. E. A systematic nomenclature for carbohydrate fragmentations in FAB-MS/MS spectra of glycoconjugates. Glycoconj. J. $1988,5,397-409$.
54. Harvey, D. J.; Wing, D. R.; Kuster, B.; Wilson, I. B. Composition of $\mathrm{N}$-linked carbohydrates from ovalbumin and co-purified glycoproteins. J. Am. Soc. Mass Spectrom. 2000, 11, 564-571.

55. Dell, A.; Morris, H. R. Glycoprotein structure determination by mass spectrometry. Science 2001, 291, 2351-2356.

56. Suzuki, N.; Khoo, K. H.; Chen, C. M.; Chen, H. C.; Lee, Y. C. N. -glycan structures of pigeon IgG: A major serum glycoprotein containing Gal $\alpha 1-4 \mathrm{Gal}$ termini. J. Biol. Chem. 2003, 278, 46293-46306.

57. Thall, A.; Galili, U. Distribution of Gal $\alpha 1-3 \mathrm{Gal} \beta 1-4 \mathrm{GlcN}$ Ac residues on secreted mammalian glycoproteins (thyroglobulin, fibrinogen, and immunoglobulin G) as measured by a sensitive solid-phase radioimmunoassay. Biochemistry 1990, 29, 3959-3965.

58. Taguchi, T.; Seko, A.; Kitaijma, K.; Muto, Y.; Inoue, S.; Khoo, K. H.; Morris, H. R.; Dell, A.; Inoue, Y. Structural studies of a novel type of pentaantennary large glycan unit in the fertilization-associated carbohydraterich glycopeptide isolated from the fertilized eggs of Oryzias latipes. J. Biol. Chem. 1994, 269, 8762-8771. 